\title{
Investigation of the Distribution and Application of Modern Chinese Psychological Verbs on Teaching Chinese as a Foreign Language
}

\section{-A Case Study of Chinese Listening, Speaking, Reading and Writing and Developing Chinese Xuan Sun}

Baicheng Normal University, Baicheng, Jilin, 137000

Keywords: psychological verbs; Chinese listening, speaking, reading and writing; Chinese development

\begin{abstract}
This paper selects Chinese listening, speaking, reading and writing, and "Chinese Development" as reference material to measure and analyze the distribution of psychological verbs in textbooks. Through the examination of the teaching materials, the combination of the psychological verbs and teaching Chinese as a foreign language is understood and analyzed.
\end{abstract}

\section{Introduction}

Vocabulary is the building material of a language. Words compose phrases into sentences, and people's free and open expression, communication and communication can be realized. The importance of vocabulary in second language teaching is self-evident. We sort out and analyze the two sets of textbooks that are widely influential in both current overseas Chinese education and domestic Chinese teaching for foreigners. We can understand and take care of psychological verbs. The Distribution and Application of Subordinates of Verbs in Teaching Chinese as a Second Language.

On the whole, the number of psychological verbs in each of the two textbooks is on the rise. From the perspective of the comparison between the new words in the textbook and the "level vocabulary for Chinese proficiency and the outline of Chinese characters", "Chinese speaking, listening, speaking, reading and writing" The choice of psychological verbs is easier than "development of Chinese", and the psychological verbs present in the two sets of teaching materials are mostly distributed in the A-level and B-level. However, at the same time, through the analysis of these two sets of textbooks, we can also see that there are some problems in the selection of new words, part of speech tags, and meaning notes of the psychological verbs. Below we will analyze in detail from these perspectives.

\section{The Selection of Words}

\subsection{Classification of mental verbs in teaching materials}

The 44 psychological verbs in the two sets of textbooks are classified as follows according to the Outline of the Chinese Standard Vocabulary and Chinese Characters:

(1) The Classification of Psychological Verbs in Chinese Speaking, Reading and Writing

Class A words: love, plan, understand, object, welcome, feel, understand, satisfied, afraid, think, agree, hope, like, believe, think, know, pay attention

Class B words: hobby, worry, remember, consider, understand, angry, familiar, hate, approve, value

C-level words: suspicion, respect

(2) Classification of psychological verbs in "Developing Chinese"

Grade A words: plan, understand, feel, thank, care, welcome, feel, understand, satisfy, think, agree, hope, like, believe, think, know, pay attention

Class B words: worried, moved, scared, hated, regretful, excited, remembered, considered, understood, hoped, angry, disappointed, familiar, hated, forgotten, envious, favored, respected 
C-level words: trust, respect

Ding level words: love

(3) Classification of all psychological verbs in two sets of teaching materials

Grade A: Love, plan, understand, object, feel, thank, care, welcome, feel, understand, satisfied, afraid, think, agree, hope, like, believe, think, know, pay attention

Class B words: hobbies, worries, emotions, fears, hate, regrets, excitement, remembering, consideration, understanding, hope, anger, disappointment, familiarity, distaste, forgetfulness, envy, approval, emphasis, respect

C-level words: suspect, trust, respect)

Ding level words: love

\subsection{Advantages of the selection of new words in textbooks}

The distribution sequence of the respective psychological verbs in the two textbooks is basically the same as the distribution trend of all the psychological verbs involved in the two textbooks. This shows that both textbooks have complied with the requirements for the quantification of Chinese vocabulary teaching. The selection and application of psychological verbs in textbooks conforms to the principles of commonality, practicability, and sequence of the vocabulary screening of the "Chinese Level Vocabulary and the Outline of Chinese Characters". This distribution is applicable to students when learning a second language. Difficult, gradual cognitive features are also consistent.

\subsection{Problems in the selection of new words in textbooks}

Different from the reference book, the outline only stipulates the level of the word and does not enumerate and explain the word meaning one by one like the Chinese reference book. This kind of quantification can lead to the lack of specificity in the specific selection of the vocabulary when the guide material is written. Sex can't clarify the need for a specific term or terms and usages for a word in a teaching phase. For example, "Thinking" and "Imagine 1" in the Dictionary of Chinese Verb Usage have the meaning "to use their brains; think about it." The general function is to use nouns, verbs, and clauses as objects, such as:

Think of problems

I was thinking of taking something to see my father.

What do you think about this plan?

In the first phase of class1, part1, Chinese reading, writing, reading and writing, the word "think" was first appeared in the fourth lesson because the content of the textbook was not readable. The part of speech and annotation we obtained through the index of the full-level 1 new word was "mv, "To think to; would like to; to think", the use of actual verbs is listed under the modal verb tagging, but the meaning and function of the word "to think" did not appear until the 15th lesson, but the textbook There is no corresponding special explanation.

\section{Part of Speech Tagging}

\subsection{Advantages of vocabulary tagging in textbooks}

Both textbooks use a lowercase English abbreviated form to mark the part of speech, which helps the students to understand the part of speech more quickly and directly. This is especially beneficial to English-speaking learners. After statistics, we found that 35 out of the 44 psychological verbs of the two sets of textbooks are consistent with the part of the outline of the outline, and they are mostly single labels, that is, only the words that appear in the class are marked. This kind of processing method is the result of the consideration and filtering of the textbook editors. It reflects the characteristics of the students' learning from easy to difficult, from simple to complex, and gradual and progressive. 


\subsection{Problems of vocabulary marking in textbooks}

In the teaching materials for foreigners, we will sometimes see the situation where the part of speech is not marked or the part of speech is inaccurate. We searched for psychological verbs found in two sets of textbooks. Many words were marked with two parts of speech in the part of speech tagging. However, there was only one kind of part of speech related usage in the text. Such as:

I plan to (v/n) go back to Beijing to see my parents during the summer vacation. (IC, level 119 class)

There are also cases where the word misplaced the standard. Such as:

The money and respect (n) are exchanged for your own sweat. ("Development of Chinese • Elementary Comprehensiveness" (II), 20 lessons)

In the same lesson, two or more parts of speech are marked at the same time in the same lesson, or the words are arbitrarily distorted regardless of the general knowledge of the reference book, reflecting that the editor was influenced by the dictionary part of speech tagging or the rigorous pursuit of the writing work. Attitudes, these practices seem to be meticulous, but they can cause students to fail to retrieve the corresponding example sentences to help understand the usage of words. The use of this example in the use of examples is ambiguous, the understanding is disturbed, and the difficulty of learning and teaching is undoubtedly added to this list. Big.

\section{The Word Notes}

\subsection{Advantages of word annotation in textbooks}

Taking "Developing Chinese" as an example, the textbook gives two or more words that are synonymous or synonymous at the same time after a new word. The mutual interpretation of word meaning makes the interpretation precise. For example, in "Development of Chinese • Elementary Comprehensiveness" (II) Lesson 2 "Fear", the interpretation is "be afraid of; fear", and "be afraid of" there is "fear" in addition to "fear" and "fear" together. With mutual restrictions on mutual meaning, students can easily grasp the meaning of the word.

From the perspective of word annotation, we think that the treatment of the new words in Chinese Reading, Writing, Reading and Writing is better, because it is its natural advantage as a textbook published in the United States. In addition to regular vocabulary comments, it also clarifies or interprets a word appearing in the text through "edge notes" or "footnotes". This can be seen as a supplement to the vocabulary notes of the new vocabulary, which is convenient for learners to understand correctly. Text, but also conducive to students to accurately grasp the lexical semantics.

\subsection{Problems in the teaching of words and annotations in textbooks}

We took "development of Chinese" as an example to examine the commentary on psychological verbs and found that in this series of textbooks, there are more similar but different mental verbs with the same meaning. Such as:

Understand: understand

Think: think, feel: think, consider

Agree; approve; approve

Respect: respect

These use cases mainly reflect the use of the same English words to annotate mental verbs that differ in semantics and grammar. This will, to a certain extent, mislead students into mixing the same or similar words into the same type and making mistakes in their use.

In the above, during the process of comparative analysis of the two sets of textbooks, taking the mental verb as a turning point as an opportunity, we have deepened our understanding of the teaching materials and provided profound thinking and inspiration for the teaching of Chinese as a foreign language. 


\section{References}

[1] Cheng Xiangwen. The Innovation of Teaching Chinese as a Foreign Language[J]. Linguistic Writing, 2005 (4): 36-42.

[2] Lu Guizhi. On the Use of New Words in English Textbooks for Teaching Chinese as a Foreign Language[J]. Journal of Hengyang Normal University, 2010, 31(1): 170-173.

[3] Wang Hanwei. On the Definition of Words in Teaching Chinese as a Foreign Language[J]. Linguistic Writing, 2009 (1): 124-133. 